\title{
Evaluation des risques des régimes fiscaux pétroliers
}

\author{
Par Emmanuel OKAMBA \\ Maître de Conférences HDR en Sciences de Gestion \\ UFR Sciences Economiques et Gestion \\ Laboratoire Institut de Recherche en Gestion \\ Université de Paris Est, Marne La Vallée \\ 5 Bld Descartes, Champs Sur Marne \\ 77454 Marne La Vallée Cedex 02 \\ okamba@univ-mlv.fr
}

\begin{abstract}
Résumé
Les contrats pétroliers entre l'Etat et les entreprises pétrolières exposent la trésorerie publique à un manque à gagner, lié au risque systémique ou à la volatilité des prix sur les marchés du pétrole, et au risque opérationnel lié aux comportements opportunistes des agents. La faible traçabilité de l'information fiscale pétrolière qui en résulte, affaiblie le reporting comptable et financier entre les parties qui ne peuvent obtenir des gains optimaux et doivent se contenter des gains satisfaisants.
\end{abstract}

\section{Mots clés}

Régime fiscal, risque, contrat pétrolier, asymétrie informationnelle, comportement opportuniste.

\begin{abstract}
Oil contracts between the state and oil companies expose the public treasury to a shortfall, linked to systemic risk or price volatility in oil markets, and to operational risk related to the opportunistic behavior of agents. The low traceability of the resulting oil tax information weakens the accounting and financial reporting between the parties, which can't obtain optimal gains and must be satisfied with the satisfactory gains.
\end{abstract}

\section{Keywords}

Tax regime, risk, oil contract, information asymmetry, opportunistic behavior. 


\section{Introduction}

Les régimes fiscaux pétroliers sont des dispositifs légaux et conventionnels par lesquels, un Etat exerce sa souveraineté sur les ressources de son sous-sol, à travers les contrats qu'il signe avec les entreprises pétrolières opérant sur son territoire. Ils visent à maximiser les recettes de l'Etat, composées des droits, taxes, impôts et redevances payées par les entreprises pétrolières compte tenu des exonérations dont ces partenaires peuvent bénéficier dans l'ensemble de la filière. Celle-ci comprend, en amont, les activités d'exploration regroupant les opérations de prospection et de recherche du pétrole, d'exploitation ou de production du pétrole brut consistant en l'appréciation, le développement des forages et la production effective. En aval, les activités logistiques ou de transport, consistant à déplacer le pétrole brut des points de production vers les points de commercialisation.

Les conventions et contrats de l'Etat avec les compagnies pétrolières, conduisent à l'attribution des permis de prospection ou de recherche et d'exploitation, en contrepartie des paiements des redevances et des impôts et taxes directes et indirectes. Les parties contractantes fournissent à l'autre le moins d'informations possibles sur les gains pétroliers pour tirer profit de leur rente informationnelle. Si le rôle du régime fiscal pétrolier est de réduire les risques que courent les parties, à travers les dispositifs d'incitation et de contrôle, dans quelle mesure, la gestion et le contrôle budgétaire des gains, permettent-ils de maîtriser les risques liés aux régimes fiscaux pétroliers ?

En situation d'asymétrie informationnelle, la maîtrise des risques liés aux régimes fiscaux pétroliers dépend du degré de traçabilité de l'information et de la pertinence du modèle d'évaluation des gains que dispose les parties. Plus le degré de traçabilité de l'information et de pertinence du modèle est élevé, plus les risques liés aux régimes fiscaux pétroliers sont faibles. Plus le degré de traçabilité de l'information et de la pertinence du modèle d'évaluation des gains est faible, plus les risques liés aux régimes fiscaux pétroliers sont élevés.

Nous voulons appliquer cette idée aux contrats pétroliers du Congo, où la fiscalité pétrolière est régie par la loi 24-54 du 23 août 1994 portant le code des hydrocarbures, le code général des impôts et le code des investissements. Ce pays a été accepté comme pays candidat à l'Initiative pour la Transparence dans les Industries Extractives (ITIE), lors de la réunion du Conseil d'Administration de l'ITIE, d'Accra, le 22 février 2008, et a été déclaré conforme par ce même Conseil, en sa session de février 2013 à Oslo. Mais cette conformité à la norme ITIE n'exclue pas l'opportunisme des parties qui cherchent à tirer profit de la rente informationnelle en rendant moins traçable les données et incertains le reporting comptable et financier et l'optimisation des gains de l'Etat.

Après avoir présenté le cadre théorique structurant les contrats pétroliers, nous analyserons les risques des régimes fiscaux pétroliers qu'encours le Congo, à travers les rapports d'audit de réconciliation des données entre l'Etat du Congo et les opérateurs pétroliers réalisé par l'ITIE en 2013, du Conciliateur Indépendant de 2010, des cabinets KPMG de 2014 et Finergies de 2016, avant de conclure.

\section{I- Le cadre théorique des régimes fiscaux pétroliers : la théorie de l'agence}

Le courant néo-institutionnelle (Gruchy (1972), Powell, DiMaggio (1991)), qui étudie les organisations comme un "nœud de contrats », place la théorie d'agence au centre de l'analyse des comportements opportunistes des agents liés par des contrats asymétriques. Il nous permet d'étudier les spécificités des contrats pétroliers, en termes d'incitations et de contrôle entre un Etat et ses cocontractants.

I-1- La théorie de l'agence : Elle s'intéresse aux relations asymétriques entre un Principal (l'Etat) et un Agent (l'entreprise pétrolière), dans lesquelles, le Principal rédige un contrat qui pousse l'Agent à faire des efforts de productivité, tout en ne lui attribuant pas une rente de monopole, afin d'éviter qu'une redevance fixe en échange de la concession, revienne à lui permettre de s'approprier de la totalité des gains de productivité. Le risque de sélection 
adverse que court alors l'Etat, le conduit à sélectionner l'entreprise la moins efficace, afin de garder sa rente informationnelle. A l'inverse, si le contrat prévoit un versement indexé sur les résultats opérationnels de l'Agent, ce dernier n'a pas intérêt à faire le moindre effort, car toute réduction du coût de production se traduira par une augmentation de la redevance versée au Principal. C'est le phénomène du risque de l'aléa moral, conduisant le Principal, dans l'exécution des contrats, à inciter des comportements inefficaces de l'Agent contractant.

Ces deux risques sont étudiés par la théorie de l'agence depuis Jensen et Meckling (1976), Fama et Jensen (1983) qui proposent des incitations et des systèmes de contrôle des comportements opportunistes dans les relations contractuelles entre un Principal et un Agent. Numa (2009) a observé dans le cadre des contrats asymétriques entre l'Etat français et les compagnies concessionnaires de Chemin de Fer privées (Agents) au XIX ${ }^{\mathrm{e}}$ siècle que, l'existence du mécanisme de partage de la garantie d'intérêt, diminuait l'incitation des Agents à l'effort. Jamet (1908), avait modélisé le calcul de cette garantie d'intérêt à partir des paramètres tels que le capital de l'Agent (C), le taux d'intérêt et d'amortissement $(t)$ correspondant à la rémunération moyenne du capital, les recettes $(\mathrm{R})$ et des dépenses d'exploitation (D). La garantie d'intérêt se justifie lorsque $\mathrm{R}<t \mathrm{C}+\mathrm{D}$. Il y a partage des bénéfices entre le Principe et l'Agent lorsque $\mathrm{R}>t \mathrm{C}+\mathrm{D}$. Le déficit d'exploitation s'écrit $t \mathrm{C}+$ $\mathrm{D}-\mathrm{R}$.

Avec la garantie d'intérêt, l'Agent fournit le Capital C, le Principal fournit chaque année la différence entre le revenu garanti $(t C)$ diminué du produit net $(\mathrm{R}-\mathrm{D})$, soit $t \mathrm{C}-(\mathrm{R}-\mathrm{D})$, ce qui donne $t \mathrm{C}+\mathrm{D}-\mathrm{R}$. Le système désintéresse l'Agent du résultat d'exploitation, car l'augmentation du produit net (R - D) diminue la part allouée par le Principal. Pour intéresser l'Agent, il faut que le Principal lui cède une fraction du revenu qui lui revient. La garantie d'intérêt correspond au partage proportionnel de la rente pétrolière ou Profit-oil, différence entre le prix de vente et le coût de revient unitaire dans les contrats de partage de la production du pétrole qui serait plus incitatif que la redevance-impôt dans les conventions pétrolières de concession.

I-2- Les spécificités des contrats pétroliers : Le problème central de tout contrat pétrolier est de définir les modalités de partage équitable de la rente pétrolière qui minimisent les risques entre le Principal et l'Agent. Le prix de transfert détermine la forme du contrat.

I-2-1- Le prix de transfert de la production entre les parties : C'est le prix du Baril de pétrole à partir duquel, le Principal et l'Agent se partagent la production. Diverses approches se distinguent pour déterminer ce prix qui est influencé par la volatilité des prix sur le marché mondial :

- Prix de Cession Officiel (PCO) par lequel, les quantités d'hydrocarbures sont valorisées en fonction d'un prix devant refléter le prix réel du marché (valeur FOB), arrêté par le Principal après concertation avec l'Agent. Ce prix fiscal négocié pour l'exercice $\mathrm{N}$, s'obtient par le rapport des coûts pétroliers engagés par l'Agent dans un champs pétrolier durant l'année N-1 sur les réserves avérées en $\mathrm{N}$ dans ce même champ ;

- moyenne pondérée des prix de ventes FOB effectuées à des tiers non affiliés à la société pétrolière durant un trimestre. Cette moyenne s'obtient par la somme des produits du prix vente unitaire par les quantités vendues divisée par la somme des quantités vendues ;

- la technique du R-factor qui consiste à déterminer les parts des parties par le rapport entre les revenus cumulés et les dépenses cumulées durant une période. Soit :

\section{R-Factor $=$ Revenus Cumulés /Dépenses cumulées}

Si : R-Factor $=1$, le Principal prend $50 \%$ de la production et l'Agent prend également 50\%, car $\mathrm{R}=1$ signifie que les sociétés ont récupéré leurs investissements, et les parties peuvent se partager le gain restant à part égale ; 
Si : R-Factor $=2$; le Principal prend $75 \%$ et 1 'Agent $25 \%$ de la production. Cela signifie qu'après la récupération du double de son investissement par l'Agent, le partage se fait cette fois-ci à l'avantage du Principal.

Quelle que soit la modalité, le contrat prévoit généralement les règles d'actualisation trimestrielle du prix de transfert. La règle la plus utilisée est l'application de l'Indice déflateur du Produit Intérieur Brut des Etats-Unis d'Amérique, tel que publié par l'OCDE dans sa revue mensuelle, à la page «National Accounts », sous les références : « National Income and product - Etats-Unis - Implicit Price Level ».

I-2-2- La typologie des contrats pétroliers : Le contrat pétrolier peut être conclu entre le Principal et un ou plusieurs Agents regroupés en un consortium sous la forme d'un jointventure, bénéficiaires des permis d'exploration et/ou d'exploitation de la concession, de l'accord de service, et du partage de production (Tableau $\mathrm{n}^{\circ} 1$ ).

Le consortium expose le Principal au risque de sélection adverse, car les entreprises les moins offrantes peuvent s'associer aux entreprises les plus offrantes et lui faire perdre, la rente informationnelle qu'il aurait gardée, en sélectionnant l'entreprise la moins efficace. Dans ce cas, les entreprises opératrices ne sont pas forcément les titulaires de permis d'exploration. Ce sont celles qui ont une forte réputation dans le métier, historiquement construite à travers leurs expériences réussies avec le Principal (Boistel (2008)). Cette réputation qui accroît la dépendance technologique et financière du Principal vis-à-vis de l'Agent, accroît également l'aléas moral du Principal, lorsque ce dernier n'a pas les moyens d'influencer les termes du contrat, et adopte le type de contrat le moins rentable que lui impose l'Agent réputé entre la concession, le partage de production et le contrat de service.

Tableau $\mathrm{n}^{\circ} 1$ : Typologie des contrats pétroliers

\begin{tabular}{|c|c|c|c|}
\hline $\begin{array}{l}\text { Part de I'État dans } \\
\text { le revenu net (hors } \\
\text { coûts pétroliers) }\end{array}$ & Régime fiscal & $\begin{array}{l}\text { Part minimale } \\
\text { de production } \\
\text { garantie à I'État }\end{array}$ & Pays concernés \\
\hline Inférieur à $\mathbf{3 0} \%$ & Concession & 0 et $10 \%$ & Irlande, Uruguay \\
\hline Entre $30 \%$ et $40 \%$ & Concession & 0 et $10 \%$ & Uruguay, US OCS, Royaume Uni \\
\hline Entre $40 \%$ et $50 \%$ & Concession, CPP & De $2 \%$ à $17,6 \%$ & $\begin{array}{l}\text { Guinée Équatoriale, Argentine, us ocs, } \\
\text { Grèce }\end{array}$ \\
\hline Entre $50 \%$ et $60 \%$ & $\begin{array}{l}\text { Concession, CPP, } \\
\text { Contrat de service }\end{array}$ & De o à $30 \%$ & US oCS, Grèce, Afrique du Sud \\
\hline Entre $60 \%$ et $70 \%$ & $\begin{array}{l}\text { Concession, CPP, } \\
\text { Contrat de service }\end{array}$ & De o à $40 \%$ & $\begin{array}{l}\text { Équateur, Pakistan II, Colombie, Gabon, } \\
\text { Maroc, Pérou, Brésil, Guatemala, } \\
\text { Mozambique, Azerbaĩdjan, Bénin, Congo, } \\
\text { Malaisie, Angola, Yémen, Indonésie, } \\
\text { Égypte }\end{array}$ \\
\hline Entre $70 \%$ et $80 \%$ & CPP, Concession & De o à $47 \%$ & $\begin{array}{l}\text { Yémen, Indonésie, Égypte, Azerbaīdjan, } \\
\text { Russie, Timor, Norvège, Tunisie, Myanmar }\end{array}$ \\
\hline Entre $\mathbf{8 0} \%$ et $90 \%$ & $\begin{array}{l}\text { Concession, CPP, } \\
\text { Contrat de service }\end{array}$ & De $12,5 \%$ à $60 \%$ & $\begin{array}{l}\text { Nigeria, Malaisie, UAE, Syrie, Indonésie, } \\
\text { Venezuela }\end{array}$ \\
\hline Supérieur à $90 \%$ & Contrat de service & De $16,7 \%$ à $30 \%$ & Venezuela, Iran \\
\hline
\end{tabular}

Cité par Ikama (2013) p.194

Dans le régime de la concession, le Principal se limite à négocier les termes du contrat, sans participer activement au développement du secteur pétrolier. C'est le régime d'impôtredevance dans lequel, l'Etat cède pour une durée déterminée ses droits de recherche ou d'exploitation à une compagnie pétrolière qui conduit et finance l'exploration à ses propres risques. Le concessionnaire a un droit exclusif d'exploitation de nature immobilière, quoique distinct de la propriété du sol. En cas de découverte d'un gisement pétrolier, le concessionnaire dispose librement, sauf disposition particulière, des productions lui revenant en fonction de sa participation au développement et récupère le pétrole après paiement d'une redevance proportionnelle et de l'impôt sur les sociétés. Soit :

Gain du Principal $\left(\mathrm{W}_{1}\right)=\mathrm{t}_{\mathrm{s}}\left(\mathrm{PCO}-\left(\frac{P V U}{2}\right)-\left(\mathrm{t}_{\mathrm{r}} * \mathrm{PCO}\right)\right)+\left(\mathrm{t}_{\mathrm{r}} * \mathrm{PCO}\right)$ 
$W=$ Part du principal en unité monétaire $;$ ts = taux d'impôt sur les sociétés $; P C O=$ Prix de vente réel unitaire; $t r=$ taux de redevance proportionnelle.

La part du Principal en volume est égale à: $\mathrm{W}_{1}=\left(\frac{W}{P V U} \times 100 \%\right)$, et celle de l'Agent s'obtient par la formule suivante: $\mathrm{W}_{2}=1-\left(\frac{W}{P V U} \times 100 \%\right)$.

Ce régime qui a été développé aux USA en 1870, s'est répondu dans le monde jusqu'à la fin de la deuxième guerre mondiale, avant d'être remplacé par les Accords de Service dans les années 1970. Ces derniers sont utilisés lorsque les coûts d'exploration et de production pétrolière sont faibles (Leenhardt (2005)). Ils prévoient que l'Agent mène la phase d'exploration et en supporte le risque. En cas de découverte d'un gisement pétrolier, il est remboursé et payé pour son service en numéraire, mais il n'a aucun droit sur la production. C'est la compagnie nationale du Principal qui assure la commercialisation de toute la production.

Quant au Contrat de Partage de Production (CPP), c'est un accord d'entreprise permettant au Principal de réduire son exposition aux risques d'exploration et d'exploitation par le partage des coûts avec le Contracteur (Agent), tout en bénéficiant des investissements en termes financiers et d'expertise indispensables pour maintenir ou accroître sa capacité de production. Ces investissements sont généralement à obsolescence programmée dont le Principal ne pourra pas utiliser durant longtemps à cause d'un transfert de technologie déficient, même si le contrat prévoit une prime destinée à la formation du personnel.

Le CPP définit le cadre d'une prestation de services et ne confère pas à son titulaire, le droit de nature immobilière. L'Agent recherche et exploite pour le compte du Principal, une substance sur un périmètre défini, en contrepartie d'une rémunération en nature. En cas de découverte de gisement de pétrole, le Contracteur est remboursé par un pourcentage du pétrole produit, représentant le «Cost-oil » ou coût pétrolier qu'il a engagé et reçoit en plus, une part égale à celle du Principal de la production restante, appelée «Profit-oil » ou profit pétrolier. Le gain du Principal $\left(\mathrm{W}_{1}\right)$ est alors composé de la moitié du Profil-oil et de la Redevance pétrolière proportionnelle. Soit :

$\mathrm{W}_{1}=50 \%\left(\mathrm{PCO}-\left(\left(\mathrm{t}_{1} * \mathrm{PCO}\right)+\left(\mathrm{t}_{2} * \mathrm{PCO}\right)\right)\right)+\left(\mathrm{t}_{2} * \mathrm{PCO}\right)$.

Avec : Profit Oil $(W) ; P C O=$ Prix de transfert réel du Baril ; le taux de remboursement des coûts pétrolier ou Cost-Stop $\left(t_{1}\right)$ et le taux de redevance minière proportionnelle $\left(t_{2}\right)$.

Le gain du Contracteur (W2) est composé de la moitié du profil-oil et du Cost-oil. Soit :

$\mathrm{W}_{2}=50 \%\left(\mathrm{PCO}-\left(\left(\mathrm{t}_{1} * \mathrm{PCO}\right)+\left(\mathrm{t}_{2} * \mathrm{PCO}\right)\right)\right)+\left(\mathrm{t}_{1} * \mathrm{PCO}\right)$.

Les CPP qui ont été inventés par l'OPEP en janvier 1961, se sont développés dans le monde depuis les années 90, et sont devenus les principaux accords dans le golfe de Guinée, où les gisements on-shore s'épuisent au profit des gisements off-shore plus coûteux, nécessitant d'importants investissements, et une gestion et un contrôle budgétaire des recettes très élaborés.

\section{II- La gestion et le contrôle budgétaire des recettes pétrolières du Congo}

Analysons le contexte économique du Congo, avant d'étudier son système de gestion et de contrôle budgétaire des recettes pétrolières.

II-1- Le contexte économique : Le pétrole représente plus de $75 \%$ des recettes au budget du Congo dans une économie exposée au risque de trésorerie publique liée à la volatilité des cours pétroliers et aux désajustements des recettes et des dépenses. En effet, dans les années 1960-70, les services représentaient 50\% du PIB du Congo, l'industrie (30\%), l'agriculture (15\%), la forêt (4\%), et les industries extractives 1\%. Depuis 1970, les industries extractives 
(le pétrole) représentent 58,6\% du PIB, les services (20,5\%), le BTP (8,3\%), l'industrie $(4,9 \%)$, l'agriculture $(3,3 \%)$ et la sylviculture $(1,4 \%)$.

Mais, dans cette économie faiblement diversifiée, les recettes pétrolières qui avaient augmenté de $13 \%$ en moyenne entre 2011 et 2013, ont baissé de $65 \%$ par rapport à 20142016 , entrainant la chute de l'ensemble des recettes publiques à $43 \%$, à cause de la forte baisse des prix du Baril du brint sur le marché mondial qui sont passés de 111,22 \$ en 2010 à moins de 43,55 \$ en 2016 quand le prix de vente du pétrole congolais est passé de 107,98\$ à $34,50 \$$ (Tableau $\mathrm{n}^{\circ} 2$ ). Cette chute des prix s'accompagne de la baisse de la production au Congo (Tableau $\mathrm{n}^{\circ} 3$ ) et de l'épuisement de ses réserves (1,6 milliard de Barils en 2011 contre 0,5 milliard prévu pour 2019). L'écart entre les prix du PCO et du Brent multiplié par les quantités vendues, donne une perte globale de 10178 554, 25 \$ durant la période étudiée.

Tableau n ${ }^{\circ} 2$ : Evolution du prix du baril du Brent depuis 2000

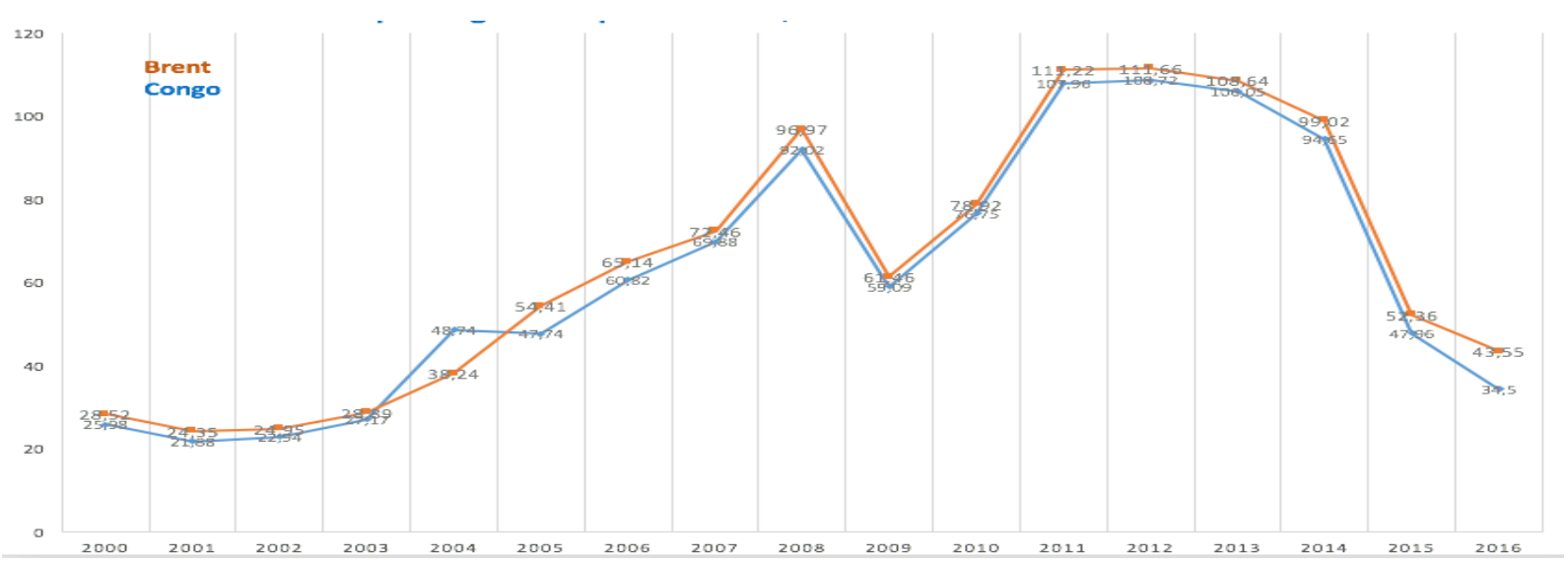

Source : D’après : http://dominiquegambier.fr/wp-content/uploads/2016/03/evolution-prix-barril.jpg

En effet, de moins d'un million de barils dans les années 60, la production pétrolière congolaise a dépassé les 100 millions de barils en 2000 pour chuter à 85 millions en 2015 . Dans le même temps, l'encours de la dette est passé de 58,1\% du PIB en 2010 à $61,5 \%$ en 2011 , puis à $45,2 \%$ en 2014 et à $57,5 \%$ en 2015 pour atteindre $120 \%$ en 2017. Selon le rapport de l'agence de notation Standard \& Poor's du 4 août 2016, le déficit budgétaire du Congo s'est élevé à $10 \%$ du PIB en 2015 contre 5,2\% en 2014, obligeant l'Etat à utiliser $40 \%$ de ses réserves déposées à la Banque des États d'Afrique Centrale (CEMAC) et $50 \%$ de ses actifs déposés en Chine. Ce déficit évoluera pour atteindre les $15 \%$ du PIB entre 2016 et 2019. L'Etat le couvrira par un emprunt obligataire de 150 milliards FCFA à 6,5\% l'an en 5 ans sur la Bourse des Valeurs de la CEMAC, et augmentera à terme, son déficit budgétaire.

Tableau n³ : Evolution de la production pétrolière depuis 2000 (en barils/j) 


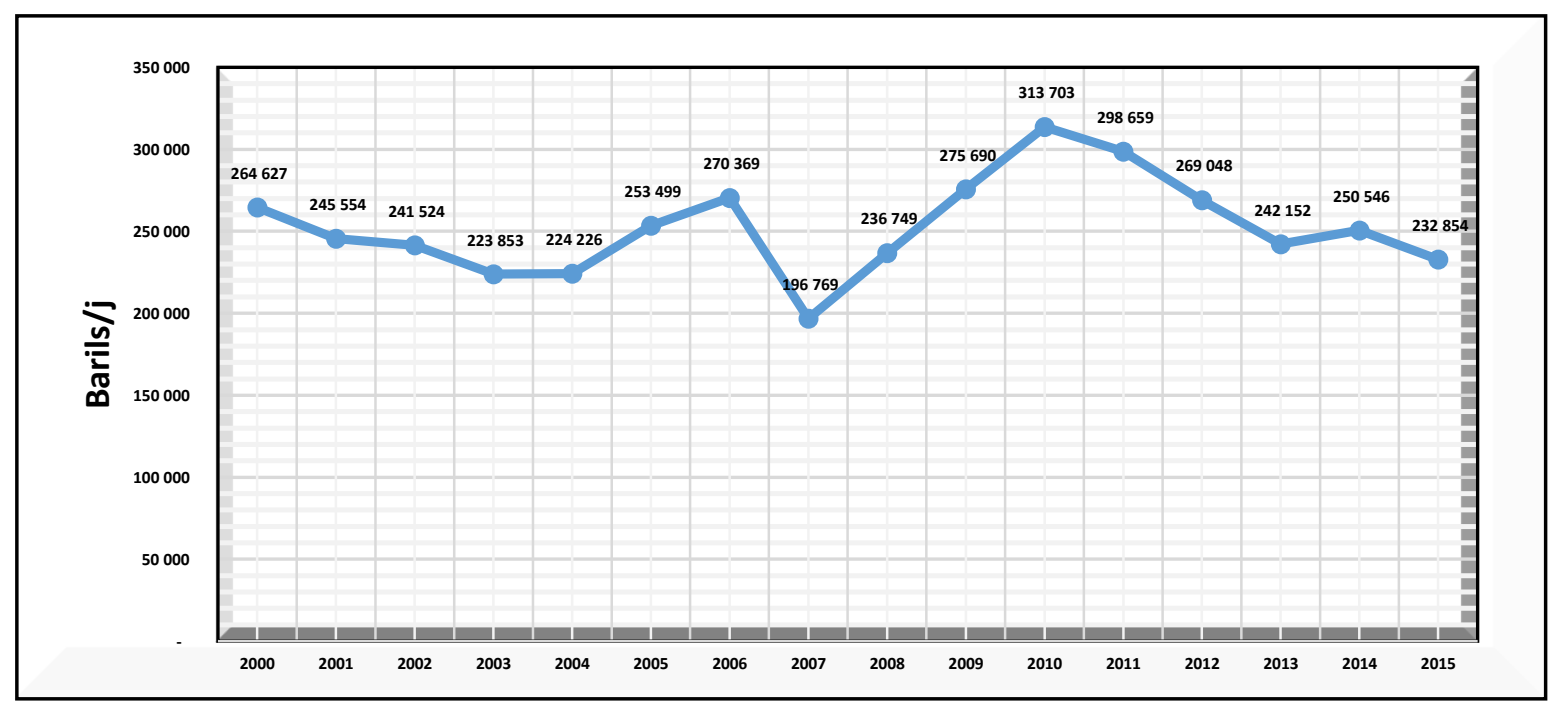

Source : D'après Ministère des Hyodrocarbures, Février 2016

Or, l'Etat recours systématique à l'emprunt gagé sur les puits de pétrole, une pratique qui augmente l'endettement par les frais de justice, les dommages et intérêts lorsque les quantités pré financées ne se réalisent. Selon 1'ONG britannique Global Witness, elle instaure un environnement " propice à la corruption et à la mauvaise gestion des fonds publics », puisqu'il est peu coûteux, ne contraint pas le Gouvernement à indiquer la manière dont-il a été contracté, ni le suivi de son utilisation. La SNPC, avait été citée devant les tribunaux des USA, sur la période 1999-2003 pour des emprunts gagés contre le pétrole d'une valeur de 650 M \$, remboursés avec 1,4 MD \$ de pétrole. Le Traider suisse Gunvor avait obtenu le droit d'exporter 20 cargaisons de pétrole brut congolais de 2 MD \$, pour un préfinancement de 750 M \$ consenti par BNP Paribas (suisse) et garanti par ce négociant. En 2016, l'Etat a gagé les emprunts chinois et des traders suisses de la SNPC contre le champ pétrolier de Moho Nord qui ne produit que $75 \%$ des quantités déjà vendues en prévisionnel.

Cependant, dans le budget 2017, les recettes ne sont que de 1.680 milliards de Francs CFA, soit une baisse de $27 \%$ par rapport aux 2.333 milliards de F CFA inscrits dans la loi de finances initiale de 2016, revue à la baisse à 2.121 milliards de FCFA à mi-juin. Ces prévisions sont fondées les prévisions de production pétrolière de 93,5 millions de barils en 2017, contre un objectif de 94,125 millions de barils en 2016. Les charges budgétaires de 2017 s'établissent à 2.107 milliards FCFA, bien en dessous 2.608 milliards FCFA de 2016. Alors qu'en mi-juin, dans la loi de finances rectificative, ce montant été ramené à 2.396 milliards FCFA. Un objectif qui ne semble pas avoir été tenu puisque ces charges se sont élevées à 2.454 milliards FCFA en 2016. Le déficit budgétaire atteindra 427 milliards FCFA en 2017.

Ainsi, le déficit budgétaire qui expose fréquemment le Trésor Public aux incidents de trésorerie, est un mauvais signal, sévèrement sanctionné par les agences de notation qui dégradent périodiquement la note souveraine du Congo. L'agence de notation américaine Standard \& Poor's (S\&P), à dégrader cette note de «B+» à «B», le 2 octobre 2015, en raison des difficultés de l'Etat à satisfaire ses obligations souveraines de longue et courte maturité. Cette sanction renchérie le coût de l'emprunt du Congo à plus de 2,5\% sur les marchés financiers internationaux. Le 4 août 2016, la même agence a dégradé la note du Congo de « B » $\mathrm{a}$ «B3», lors que la gestion des régimes fiscaux pétroliers est problématiques.

II-2- Les régimes fiscaux pétroliers : Plus de 24 principales entreprises, publiques et privées interviennent aussi bien en amont qu'en aval de la filière pétrolière congolaise, dont les plus 
importantes sont : Total E\&P Congo (56\% de la production pétrolière du Congo), ENI-Congo (26\%), Parenco (11\%), CMS Nomeco (4\%) et autres (3\%) (Tableau n $\left.{ }^{\circ} 4\right)$.

Tableau $n^{\circ} 4$ : Principaux opérateurs pétroliers du Congo en 2016

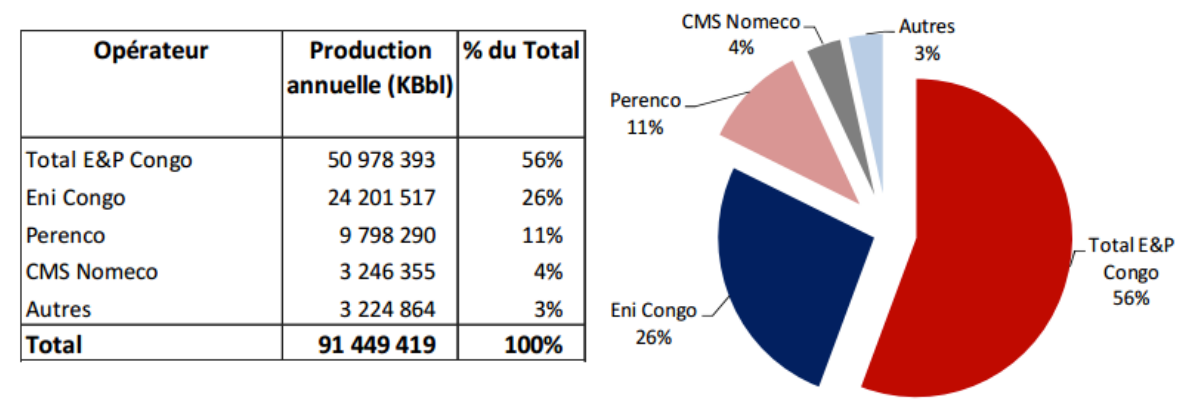

Source : Rapport ITIE-congo, 2016

Parmi ces entreprises, 14 sont titulaires de 33 permis d'exploration dont les plus importantes sont Total E\&P Congo $(9,09 \%)$ et ENI-Congo (6,06\%). Seulement 8 entreprises sont titulaires de 35 permis d'exploitation dont $40 \%$ pour Total E\&P Congo, $40 \%$ pour ENI-Congo, 5,71\% pour Congorep et par 14,29\% pour d'autres exploitants. La Société Nationale des Pétroles du Congo (SNPC) qui explore et exploite le pétrole, prend des participations dans $35 \%$ des entreprises, contre ENI-Congo (24\%), Buren (14\%), Total-Congo (10\%) et autres (17\%).

Le Congo produit principalement trois types de pétroles dont le Djéno (74\%) produit peu sulfuré avec une densité moyenne, le Nkossa (20\%), pétrole léger et peu sulfuré et le Yombo (4\%), pétrole lourd et très sulfuré (Tableau $\left.\mathrm{n}^{\circ} 5\right)$.

Tableau $n^{\circ} 5$ : Types de pétroles congolais en 2016

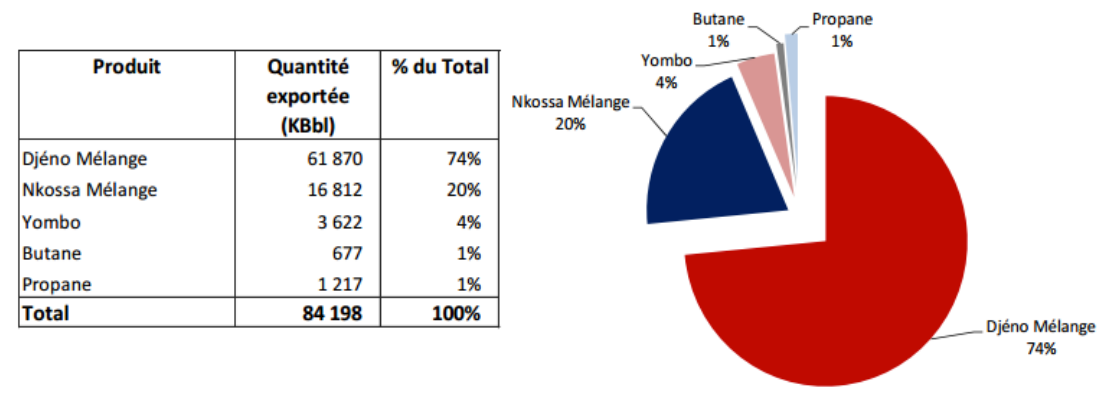

Source : Rapport ITIE-Congo, 2016

Deux principaux régimes fiscaux se distinguent (Tableau $\left.{ }^{\circ} 6\right)$ :

- la concession ou l'impôt-redevance est le régime le plus ancien, dont le premier contrat remonte à 1949, avec l'attribution du permis de recherche pétrolière par l'administration coloniale française à la Société des Pétroles de l'Afrique Equatoriale Française (SPAEF) pour 40 ans. Le 31 mai 1960, la première concession Pointe-Indienne $\left(10,76 \mathrm{~km}^{2}\right)$, fut attribuée à la SPAEF pour 75 ans. En 1968, le permis de recherche Pointe-Noire Grands Fonds (4100 $\mathrm{km}^{2}$ ), est attribué à l'Entreprise de Recherche et d'Activités Pétrolières (ERAP), créée en 1965 en France, devenue Elf Aquitaine en 1969, et absorbée par Total en 2010. Le permis de recherche Madingo maritime $\left(415 \mathrm{~km}^{2}\right)$ est octroyé à AGIP S.A. (italienne). En 1969, les filiales cocontractantes Elf-Congo et Agip-Congo découvrent 600 millions de tonnes de réserves de pétrole dans le champ Emeraude.

Pour assurer sa souveraineté, sous l'effet des chocs pétroliers, le Congo créa, en 1973, la société Nationale de Recherches et d'Exploitation Pétrolière (Hydro-Congo), liquidée après sa faillite et remplacée par la Société Nationale des Pétroles Congolais (SNPC) en avril 1998. En 
1977, le permis Mer Profonde est attribué à la société Getty-oil qui fore en 1978 le puits Sangha-Marine I.

A partir de 1994, la recherche par Grand Fond et l'utilisation de nouvelles technologies permettent d'entreprendre l'exploitation et le développement des gisements sous une grande profondeur d'eau. Elf-Congo découvre les gisements Kombi, Tchibouéla-Est et Litanzi, quand Kitina et Ikalou-Sud sont mis en valeur par Agip et Yombo par Amoco devenue CMSNomeco-Congo. D'autres gisements seront découverts par la suite tels que: Djambala, Sounda, Loussima, Tchibéli et Emeraude-Nord. Le gisement de Nkossa, dont la durée d'exploitation est estimée à 30 ans, est le plus important de tous, avec une production globale de près de 5 millions de tonnes de pétrole par an et de 300 tonnes de gaz de pétrole liquéfié.

En 2010, 20,69\% des contrats pétroliers du Congo étaient sous le régime de la Concession contre seulement $3,03 \%$ en 2016 ; alors que les contrats de partage de la production qui représentaient 79,31\% des contrats en 2010 sont passés à 96,97\% en 2016 ;

- le Contrat de Partage de la Production permet au Principal de partager la production avec un Contractant, après déduction des coûts engagés par ce partenaire. Les termes du CPP sont : le Profit-oil (W) ; le Prix de transfert négocié par Baril de pétrole (PCO); le taux de remboursement des coûts pétroliers engagés par l'Agent ou Cost-Stop ( $\mathrm{t}_{1}$ ) et le taux de redevance minière proportionnelle $\left(\mathrm{t}_{2}\right)$. La part de pétrole du Principal est égale à :

$\mathrm{W}=50 \%\left(\mathrm{PCO}-\left(\left(\mathrm{t}_{1} * \mathrm{PCO}\right)+\left(\mathrm{t}_{2} * \mathrm{PCO}\right)\right)\right)+\left(\mathrm{t}_{2} * \mathrm{PCO}\right)$.

A cette part, il y a lieu d'ajouter deux incitations :

Tableau n ${ }^{\circ} 6$ : Typologie des contrats pétroliers du Congo en 2010

\begin{tabular}{|c|c|c|c|c|c|c|c|c|c|c|c|}
\hline \multirow{2}{*}{$\begin{array}{l}\text { Permis de } \\
\text { Recherche }\end{array}$} & \multirow{2}{*}{$\begin{array}{l}\text { Permis d'Exploitation } \\
\text { et concession }\end{array}$} & \multirow{2}{*}{ Superficie } & \multirow{2}{*}{ Textes attributifs } & \multirow{2}{*}{\begin{tabular}{|l} 
Origine \\
de validité
\end{tabular}} & \multirow{2}{*}{ Fin de validitít } & \multirow{2}{*}{ Titulaire } & \multicolumn{4}{|c|}{ Participatic \% } & \multirow{2}{*}{\begin{tabular}{|l} 
Année de \\
production
\end{tabular}} \\
\hline & & & & & & & associé & & opérateur & & \\
\hline & Emeraude (C) & & $\begin{array}{l}\text { Décret n70-354 } \\
\text { du } 18 \text { novermbre } 1970\end{array}$ & 18 mai 1970 & 17 nov, 2020 & Congorep & SNPC & 49 & congorep & 51 & 1971 \\
\hline \multirow[t]{5}{*}{ Ex PNGF } & Louango Ouest (C) & 65,091 & \begin{tabular}{|l|} 
Décret n’73-168 \\
du 21mai 1973
\end{tabular} & 21 mai 1973 & 21 mai 2023 & Tep Congo & ENI Congo & 35 & Tep Congo & 65 & 1977 \\
\hline & Likouala (C) & 63,45 & $\begin{array}{l}\text { Décret no78-416 } \\
\text { du } 27 \text { mai } 1978\end{array}$ & 27 mai 1978 & 27 mai 2028 & Likouala SA & ENI Congo & 35 & Likouala SA & 65 & 1980 \\
\hline & Yanga- sendj ( $\mathrm{C}$ ) & 170 & $\begin{array}{l}\text { Décret no79-659 } \\
\text { du } 1 \text { déc } 1979\end{array}$ & 1 décembre 1979 & 1 décembre 2029 & Tep Congo & $\begin{array}{l}\text { ENI Congo } \\
\text { RC 15\% }\end{array}$ & $29,75 \%$ & Tep Congo & 55,25 & 1981 \\
\hline & Tchibouela $(P, E)$ & 135 & \begin{tabular}{|l|} 
Décret no ${ }^{\circ} 85-883$ \\
du 8 jullet 1985
\end{tabular} & 3 juilet 2010 & 8 juilet 2015 & Tep Congo & ENI Congo & 35 & Tep Congo & 65 & 1987 \\
\hline & Tchendo $(\mathrm{P}, \mathrm{E})$ & 82 & \begin{tabular}{|l|} 
Décret no ${ }^{\circ} 88-569$ \\
du 30 jullet 1988
\end{tabular} & 30 iuilet 2008 & 30 iu illet 2013 & Tep Congo & ENI Congo & $35 \%$ & Tep Congo & 65 & 1991 \\
\hline \multirow[t]{2}{*}{ Ex Madingo } & Loango-est (C) & 50,67 & \begin{tabular}{|l|} 
Décret not73-169 \\
du 21 mai 1973
\end{tabular} & 21 ma i 1973 & 21 mai 2023 & ENI Congo & Tep Congo & $35 \%$ & ENI Congo & & 1988 \\
\hline & Zatchi $(P, E)$ & 77,5 & \begin{tabular}{|l|} 
Décret no86-745 \\
du 03 juin 1986
\end{tabular} & 3 juin 1986 & 3 juin 2011 & ENI Congo & Tep Congo & 35 & ENI Congo & 65 & 1988 \\
\hline \multirow[t]{3}{*}{ Ex haute Mer } & Nkossa $(P, E)$ & 100,46 & $\begin{array}{l}\text { Décret no } 92-323 \\
\text { du } 24 \text { juin } 1992\end{array}$ & 24 juin 1992 & 24 juin 2022 & Tep Congo & $\begin{array}{l}\text { Chevron } \\
\text { SNPC } \\
\end{array}$ & $\begin{array}{r}31,5 \% \\
15 \% \\
\end{array}$ & Tep Congo & $53,5 \%$ & 1996 \\
\hline & Nsoko $(P, E)$ & 40,1 & $\begin{array}{l}\text { Décret n²003-127 } \\
\text { du } 26 \text { juillet } 2003\end{array}$ & 26 juilet 2003 & 26 juilet 2013 & Tep Congo & $\begin{array}{l}\text { Chevron } \\
\text { SNPC }\end{array}$ & $\begin{array}{l}30 \% \\
15 \%\end{array}$ & Tep Congo & $51 \%$ & $\begin{array}{l}\text { en } \\
\text { développernent }\end{array}$ \\
\hline & & & & & & & ENGEN & $\%$ & & & \\
\hline Ex Marine VII & Kitina $(P, E)$ & 92,87 & \begin{tabular}{|l|} 
Décret $n^{\circ} 94-285$ \\
du 21 aoôt 1994
\end{tabular} & $21 \mathrm{ju}$ in 1999 & 21 juin 2024 & ENI Congo & \begin{tabular}{|l|} 
SNPC \\
Chevron
\end{tabular} & $\begin{array}{l}35 \\
29,25\end{array}$ & ENI Congo & 35,75 & 1997 \\
\hline \multirow[t]{3}{*}{ Ex Marine VI } & Djambala (P,E) & 39,44 & \begin{tabular}{|l|} 
Décret no97-87 \\
du 10 avril 1997
\end{tabular} & 10 av rì 2007 & 10 avril 2012 & ENI Congo & SNPC & 35 & ENI Congo & 65 & 1999 \\
\hline & Foukanda (P,E) & 227,18 & Décret no98-274 & 24 juilet 1998 & 24 jullet 2013 & ENI Congo & SNPC & 35 & ENI Congo & 65 & 2001 \\
\hline & Mwafi $(P, E)$ & 185,38 & $\begin{array}{l}\text { Décret no99-274 } \\
\text { du } 09 \text { jullet } 1999 \\
\end{array}$ & 9 juilet 1999 & 9 ju ilet 2014 & ENI Congo & SNPC & 35 & ENI Congo & & 2001 \\
\hline \multirow[t]{3}{*}{ kouilou } & kouakouala $(P, E)$ & $\overline{162,1}$ & $\begin{array}{l}\text { Décret n'97-67 } \\
\text { du } 04 \text { avril 1997 } \\
\text { mod Décret n²000-31 } \\
\text { du } 18 \text { mars } 2000\end{array}$ & 10-janv-08 & 10 janvier 2013 & ENICOngo & BUREN & & $\begin{array}{l}\text { ENI Congo } \\
\text { SNPC }\end{array}$ & & 2000 \\
\hline & Mboundi $(P, E)$ & 145,5 & Décret n'2002-248 & 15 juilet 2002 & 15 jullet 2012 & ENI Congo & \begin{tabular}{|l|} 
Buren \\
TULLOW \\
\end{tabular} & & ENI Congo & & 2002 \\
\hline & & & du 15 jullet 2002 & & & congo & SNPC & & & & \\
\hline
\end{tabular}




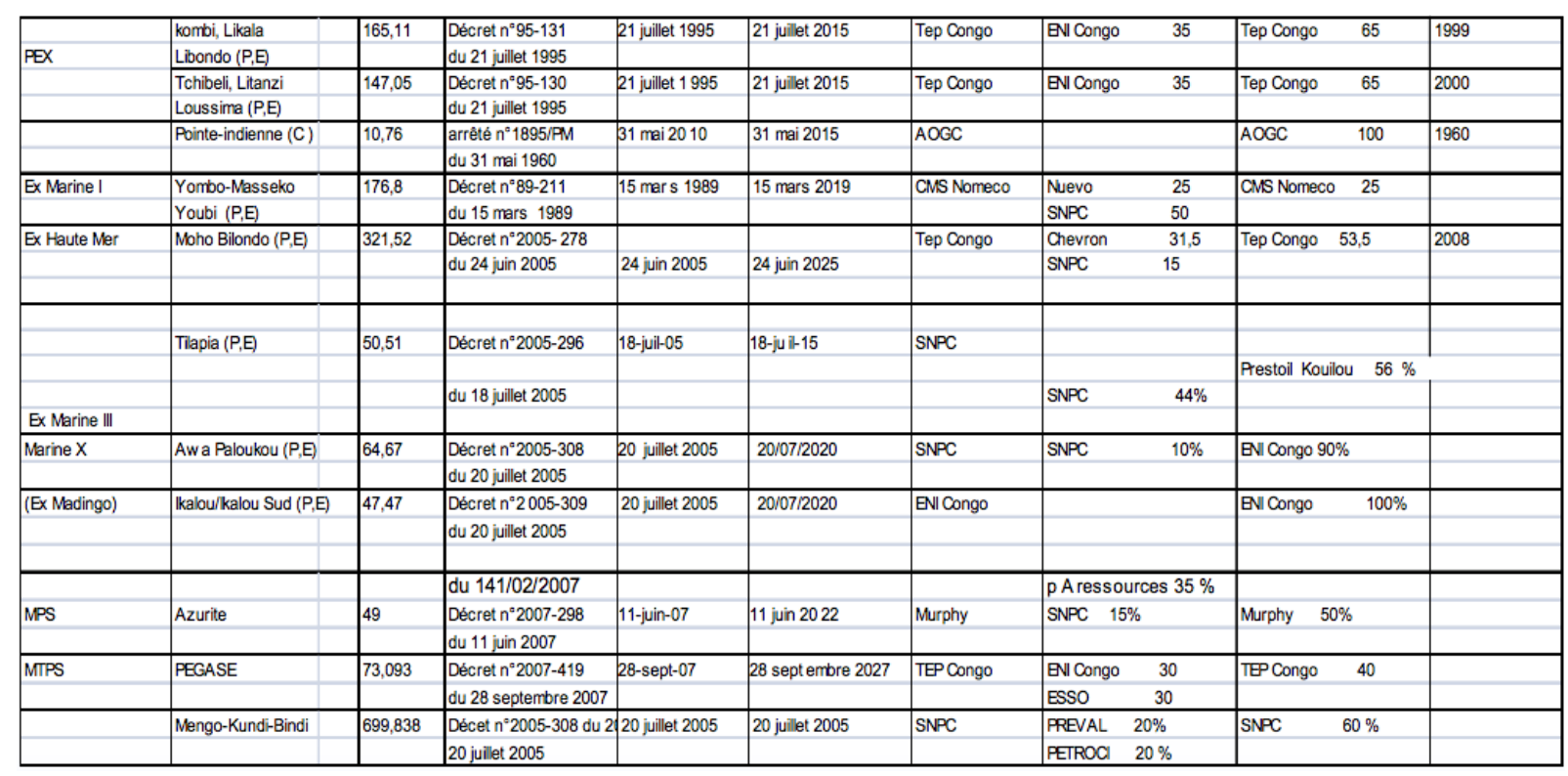

Source : Rapport du Conciliateur Indépendant, 2010

- l'Excess-oil et le gain au-dessus du seuil de prix haut (22\$/Baril), quand le Cost-stop qui assure le remboursement des coûts pétroliers devient excédentaire après déduction de ces coûts. Cet excédent est partagé à part égale entre le Principal et l'Agent. Soit :

$\mathrm{E}=50 \%\left(\frac{P V p}{2}\right)-\left(\mathrm{PVP} * \mathrm{t}_{1}\right)-\left(\mathrm{PVP} * \mathrm{t}_{2}\right)$

$E=$ Excess-oil $; P V P=$ Seuil du prix haut $; t_{1}=$ taux de redevance proportionnelle,$t_{2}=$ taux de provision d'investissements diversifiés.

- le super Excess-oil défini comme la différence entre la production nette valorisée au prix fixé et cette même production nette valorisée au prix haut. Si le prix est compris entre 10 et 14 $\$$, la part de chaque partie est multipliée par $7 \$ /$ Baril. Si ce prix est inférieur à $10 \$$, chaque part est multipliée par le $7 / 10^{\mathrm{e}}$ du prix. Lorsque le prix réel (PCO) est supérieur au prix de négociation prévu ( $\mathrm{PVP}=22 \$ /$ Baril), apparaît le chiffre d'affaires au-dessus du seuil de prix haut. C'est le super Excess qui est partagé entre les parties à $66 \%$ pour le Principal et à $34 \%$, pour l'Agent. La part du Principal s'obtient de la manière suivante :

$$
\mathrm{C}=66 \%\left((\mathrm{PCO}-\mathrm{PVP})-\left(\mathrm{PCO} * \mathrm{t}_{1}-\mathrm{PVP} * \mathrm{t}_{1}\right)-\left(\mathrm{PCO} * \mathrm{t}_{2}-\mathrm{PVP}^{*} \mathrm{t}_{2}\right) .\right.
$$

Le gain du Principal par Baril est alors égal à $\mathrm{W}_{1}: \mathrm{W}+\mathrm{C}+\mathrm{E}$, et celui de l'Agent est égal à $\mathrm{W}_{2}=\mathrm{PCO}-(\mathrm{W}+\mathrm{C}+\mathrm{E})$.

Le gain du Principal en volume est obtenu par le rapport : $\frac{W+C+E}{P V R} \times 100$; et celui de l'agent

s'en déduit par : $1-\left(\frac{W+C+E}{P V R} \times 100\right)$.

Le seuil de 22\$/Baril est déterminé depuis le premier janvier 1995 et son actualisation trimestrielle par application de l'indice déflateur du Produit Intérieur Brut des Etats-Unis d'Amérique, s'effectue par la formule suivante :

Seuil Prix Haut ${ }_{n}=$ Seuil de prix au $1^{\text {er }}$ janvier de l'année de départ du puits $\mathrm{x}$ Indice Prix PIB $\mathrm{USA}_{\mathrm{n}-1} /$ Indice valorisé du trimestre.

Pour le seuil haut de novembre 2016, par exemple, pour les Zones de Permis Pex TchibeliLitanzi/haute Mer A, le seuil de 22\$/Baril au $1^{\text {er }}$ janvier 1994, indice départ au $4^{\mathrm{e}}$ trimestre 1994 (Q4) 89,00 (Base 2000), 79,3 (Nouvelle Base 2005) indice corrigé 72,06 arrondi à 72.1 
(Base 1010), indice déflateur du PIB des USA 107.3 pour le premier trimestre 2015, nous aurons :

Seuil Prix Haut en novembre $2016=22 * 107 / 72,06=32,07 \$$.

Le prix fiscal négocié annuel moyen entre les membres du Comité de Gestion est inférieur au prix du marché. Il est en chute libre en passant de 94,66\$/Baril en 2014 à 47,64 en 2015 et à 34,52 en 2016. Pour le Djeno, ce prix est passé respectivement de 94,52 à 46,47 en 2014 et à 39,34\$/Baril en 2016. Pour le Nkossa, ce prix est passé de 98,32 à 51,47 et à 43,56 \$/Baril. Le Yombo (5\%), ce prix est passé de 88,35 à 43,711 et à $31,47 \$ /$ Baril. Le prix de vente des cargaisons de l'Etat se situe en dessous de ce prix fiscal, et génère mécaniquement une perte estimée pour les deux régimes fiscaux pétroliers à 1,65 \$/Baril, soit pour les productions nettes des champs évaluées à 390 millions de barils, environ plus de 600 millions \$. Cette perte est amplifiée par les comportements délétères des agents et les décalages des délais, liés à la faible formalisation et transmission des informations fiables du Contracteur vers l'Etat, engendrant une évaluation tardive des écarts entre les prévisions et les réalisations des recettes, et de leur régulation.

Or, chaque prix de type de pétrole reflète la valeur de la qualité d'hydrocarbures liquides, FOB terminal de chargement au Congo, sur le marché international. Il est déterminé paritairement en dollars par Baril par mois. Les entités composant le Contracteur communiquent aux autorités compétentes du Congo les informations nécessaires sur la production et les coûts dans le mois suivant la fin de chaque trimestre, lors de leur rencontre afin de déterminer d'un commun accord, pour chaque qualité d'hydrocarbures liquides produite, le prix fixé pour chaque mois du trimestre écoulé. En 2016, en suivant les règles de partage de la production, le PCO du Pétrole Djeno n'a été favorable au Principal qu'à partir de mai, quand ce prix est devenu supérieur à $39,45 \$$ et la part de ses gains a atteint les $50 \%$ du PCO, soient 19,73\$ équivalents aux $60 \%$ des coûts pétroliers récupérés par l'Agent. Alors que la part de ce dernier équivaut également à $50 \%$ du $\mathrm{PCO}$, soient $19,73 \$$, majorée de 8,73\$ $\left(22,11 \%\right.$ du PCO), représentants l'Excess $(3,74 \$)$ et le super Excess $(4,98 \$)$ (Tableau n $\left.{ }^{\circ}\right)$.

Tableau $\mathrm{n}^{\circ} 7$ : Résultats de la simulation du modèle de partage de la Production à partir du pétrole de Djéno au PCO de 39,45\$/Baril et au Prix Prévisionnel de 22\$/Baril

\begin{tabular}{|c|c|c|c|c|c|c|c|c|}
\hline Eléments & Formule & Quantité & Taux & Montant & \multicolumn{2}{|l|}{$\begin{array}{c}\text { Part } \\
\text { Principal } \\
\end{array}$} & \multicolumn{2}{|l|}{$\begin{array}{c}\text { Part } \\
\text { Agent }\end{array}$} \\
\hline Coût Pétrolier (Cost-oil) & $\mathrm{PCO} * \mathrm{t} 1$ & 39,45 & 0,50 & 19,73 & 5,92 & $15,00 \%$ & \multirow[t]{2}{*}{19,73} & \\
\hline Provision Investissement Diversifié (PID) (I) & $\mathrm{PCO} * \mathrm{t} 3$ & 39,45 & 0,01 & 0,39 & 0,39 & $1,00 \%$ & & \\
\hline Total Production Nette (Profit-oil) (1) & $=\mid-I I I-I I I$ & & & 26,04 & 6,31 & $16,00 \%$ & 19,73 & $50,00 \%$ \\
\hline Coût Pétrolier (Cost-oil) (b) & Prix Prévisionnel*t1 & 22,00 & 0,50 & 11,00 & $50 \%$ & & $50 \%$ & \\
\hline Total Excess (2) & $=P-a-b-c$ & & & 7,48 & 3,74 & $9,48 \%$ & 3,74 & $9,48 \%$ \\
\hline Ecart de Prix $\left(p^{\prime}\right)$ & PCO-Prix Prévisionnel & 39,45 & 22,00 & 17,45 & \multirow{3}{*}{$66 \%$} & & \multirow{3}{*}{$34 \%$} & \\
\hline Ecart de Redevance Minière Prop.(a') & $\begin{array}{l}\text { - (Coût Pétrolier (I)- Coût } \\
\text { Pétrolier (a)) }\end{array}$ & 5,92 & 3,30 & 2,62 & & & & \\
\hline Ecart PID (c') & -(PID (I)-PID(a)) & 0,39 & 0,22 & 0,17 & & & & \\
\hline Total Super Excess ( 3 ) & $=P^{\prime}-a^{\prime}-b^{\prime}-c^{\prime}$ & & & 14,66 & 9,67 & $24,52 \%$ & 4,98 & $12,63 \%$ \\
\hline \multirow{2}{*}{\multicolumn{3}{|c|}{ Ventilation de chaque part }} & \multicolumn{2}{|c|}{ Coût Pétrolier (60\%) } & & & $50,00 \%$ & \\
\hline & & & \multicolumn{2}{|l|}{ Total } & $50,00 \%$ & & $72,11 \%$ & \\
\hline
\end{tabular}

De janvier à avril, le PCO étant inférieur à 39,45\$/Baril, la part du Principal hors la somme des Excess est inférieure à celle de l'Agent sans que le contrôle à posteriori exercé par le Comité de Gestion n'ait corrigé cette situation. Ici, le Principal doit à l'Agent l'écart entre les 
gains qu'il perçoit et la part des gains revenant à l'Agent, soit 8,72 \$, environ $22,11 \%$ des gains du Principal. La technique de contrôle apostériori des écarts adoptée par les parties, accentue donc les pertes du Principal.

Ces résultats sont voisins de ceux obtenus par Lamotte et Porcher (2011) qui ont comparé les règles de partage de la rente pétrolière entre la convention de concession et le contrat de partage de production du Congo au $\mathrm{PCO} / \mathrm{baril}$ compris entre 0 et $120 \$$ pour un prix prévisionnel de 26 \$/Baril et un taux de redevance minière proportionnelle de $12 \%$ (Tableau $\left.n^{\circ} 9\right)$.

Tableau $n^{\circ} 8$ : Simulation des contrats pétroliers

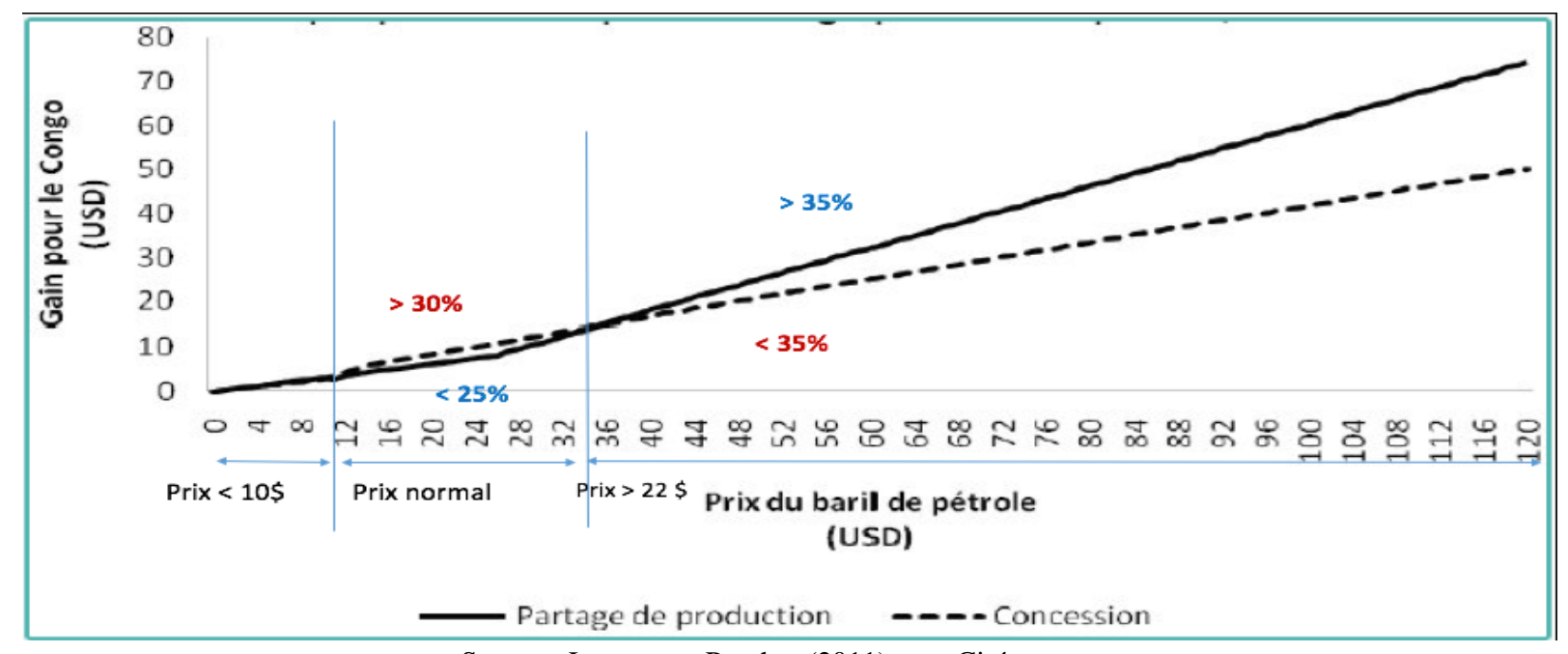

Source : Lamotte et Porcher (2011), opt. Cité

Ils en conclurent que le régime de concession était plus favorable au Principal que le régime de partage de production à l'intérieur de la zone de prix comprise entre 15 et $35 \$ / \mathrm{Baril}$. Audelà de $35 \$$, le régime de partage de production est plus favorable pour le Principal. Pour eux : «Le Congo est donc, sous le régime de partage de production, presque toujours perdant car le prix du baril varie toujours entre 20 et $30 \$$ à cause de l'OPEP. Lorsque le prix du baril passe en dessous de 20\$, l'OPEP ferme ses robinets pour que le prix remonte et inversement lorsque le prix dépasse 30\$, l'OPEP ouvre ses robinets pour que les prix descendent. Le changement de régime de la loi du 23 août 1994 a donc fait perdre beaucoup d'argent au Congo ».

L'évolution des prix des pétroles en 2016 montre une situation particulièrement précaire pour le Principal (Tableau n ${ }^{\circ}$ ), notamment les prix de Yambo (sous le régime de la Concession). En février 2016, le PCO moyen des pétroles congolais est de 26,304\$/Baril quand le prix moyen du marché international est de 33,500\$/Baril. Les gains des parties ne sont optimaux que si l'information sur les coûts de revient et les prix de vente est traçable, transparente et circule dans les délais impartis entre le Principal et l'Agent et que les parties disposent du même modèle d'évaluation de la rente.

Tableau ${ }^{\circ} 9$ : Evolution des prix de vente du Baril du pétrole congolais en 2016

\begin{tabular}{|l|c|c|c|c|c|c|c|c|c|c|c|c|c|}
\hline & JANVIER & FEVRIER & MARS & AVRIL & MAI & JUIN & JUIL. & AOUT & SEPT. & OCT. & NOV. & DEC. & Moyenne \\
\hline PRIX DJENO & 25,455 & 27,370 & 34,090 & 39,257 & 43,126 & 44,687 & 39,547 & 43,674 & 43,802 & 46,315 & 42,372 & 42,372 & 39,339 \\
\hline PRIX N'KOSSA & 30,464 & 31,744 & 38,376 & 41,832 & 48,553 & 50,630 & 43,408 & 48,495 & 47,019 & 50,012 & 46,069 & 46,069 & 43,556 \\
\hline PRIX YOMBO & 18,014 & 19,799 & 26,450 & 29,442 & 34,835 & 36,299 & 34,301 & 34,972 & 35,869 & 35,869 & 35,869 & 35,869 & 31,466 \\
\hline PRIX NEMBA & ND & ND & ND & 44,628 & 44,628 & 44,628 & ND & ND & ND & ND & ND & ND & \\
\hline PRIX BUTANE & 28,912 & 28,184 & 31,278 & 28,664 & 27,974 & 33,024 & 30,394 & 31,503 & 37,481 & 39,842 & 36,318 & 36,318 & 32,491 \\
\hline PRIX PROPANE & 15,985 & 17,834 & 14,274 & 14,427 & 17,481 & 16,197 & 15,920 & 14,771 & 16,967 & 20,363 & 19,320 & 19,320 & 16,905 \\
\hline
\end{tabular}




\section{III- Procédure de gestion et de contrôle budgétaire des recettes pétrolières du Principal}

L'établissement du budget des recettes pétrolières du Principal suit une procédure dont la faible traçabilité pose le problème du reporting comptable et financier entre le Contracteur et les services du Principal (Ministères des Hydrocarbures, Direction Générale des Impôts, Direction Générale du Trésor), et de l'optimisation des gains des parties.

III-1- Elaboration du budget : Le CCP impose à l'Agent, l'établissement d'un plan de Travaux Pétroliers et d'un budget global qui doivent être approuvés par le Comité de Gestion au plus tard le 15 Décembre de chaque année civile. A la même date, le Comité de Gestion approuve également le montant de la provision que le Contracteur constitue pour chaque Baril d'hydrocarbures liquides restant à produire. Chaque entité membre du Contracteur imputera en conséquence sur les Coûts Pétroliers de chacune des années civiles suivantes, une somme égale au montant de la provision à constituer par Baril restant à produire multipliée par la part de la production d'hydrocarbures liquides lui revenant au titre de l'année civile considérée sur la concession ou le permis d'exploitation concerné.

En cas de besoin, au plus tard le 15 novembre de chaque année civile, le Contracteur présentera au Principal, les modifications qu'il convient d'apporter à l'estimation des réserves restant à exploiter et au coût des Travaux d'Abandon prévus. En fonction de ces nouvelles estimations de réserves restant à produire et des nouvelles estimations de coûts des Travaux d'Abandon, le Contracteur déterminera le cas échéant, compte tenu des provisions déjà effectuées à ce titre, le nouveau montant en Dollars des provisions à constituer pour l'ensemble des années civiles à venir jusqu'à l'arrêt de la production sur chaque Baril d'hydrocarbures liquides qui sera produit. Le Comité de Gestion approuvera ce nouveau montant, le 15 Décembre de la même année au plus tard.

Lorsque le Contracteur estime qu'au total $75 \%$ des réserves prouvées d'une concession ou d'un permis d'exploitation objet du Contrat devraient avoir été produites au cours de l'année civile qui suivra, il soumettra au Principal, pour le compte du Contractant pour, au plus tard le 15 novembre de l'année civile en cours, le Programme de Travaux d'Abandon qu'il se propose de réaliser sur cette concession ou ce permis d'exploitation avec un plan de remise en état du site, un calendrier des travaux prévus et une estimation détaillée de l'ensemble des coûts liés à ces Travaux d'Abandon.

Le Comité de Gestion est composé d'un représentant du Contracteur et d'un représentant du Congo. Chacun d'eux à un suppléant. Il examine les Programmes de Travaux et les Budgets qui font l'objet d'une approbation à l'unanimité et contrôle leur exécution. Il se réunit au moins deux fois par an pour discuter et approuver le Programme de Travaux et le Budget et pour entendre le rapport du Contracteur sur l'exécution du budget afférent à l'année civile précédente.

Il peut se réunir chaque fois que le Contracteur le demande, sur convocation adressée 15 jours à l'avance. La convocation contient l'ordre du jour proposé, la date, l'heure et le lieu de la réunion. Le Contracteur fait parvenir au Principal les éléments d'information nécessaires à la prise des décisions figurant à l'ordre du jour 8 jours avant la réunion. Le Principal peut à tout moment demander que le Contracteur convoque une réunion pour délibérer sur des questions déterminées qui font alors partie de l'ordre du jour de la réunion.

Le Contracteur prépare un procès-verbal écrit de chaque séance et en envoie une copie au Congo dans les 15 jours de la date de la réunion, pour approbation ou remarques dans les trente jours à compter de la date de réception.

III- 2- Le contrôle budgétaire : Le Contracteur présente au Principal, dans un délai de 60 jours à compter de la date d'effet, l'état des Travaux Pétroliers réalisés sur les Permis à la Date d'Effet ainsi que le Programme de Travaux décidé pour l'année civile en cours, avec le budget correspondant. Dans les 90 jours suivant la fin d'une année civile ou, en cas de fin du Contrat, dans les trois mois de cette expiration, le Contracteur rend compte au Principal de la façon dont a été exécuté le Budget afférent à l'année civile écoulée. 
Le contrat de partage de production de 1994, dispose que les hydrocarbures liquides produits dans le cadre d'une zone de permis deviennent la propriété indivise du Congo et l'Agent. Les parts de propriétés ne sont divisées qu'à la sortie des installations de stockage dans le cas d'une expédition par navire pétrolier. Le point de transfert de propriété est le point de raccordement entre le navire et les installations de chargement. Le Principal ne devient propriétaire de sa part de pétrole que lorsque la société nationale en prend livraison et quand l'Etat l'autorise sa commercialisation. La SNPC, faute de pouvoir re-stocker d'importantes quantités de pétrole et d'en subir les coûts de transbordement, de stockage, de nouveau raccordement, ne peut maîtriser le plan des ventes établie par chaque Agent, alors qu'elle effectue des ventes à terme, des ventes futures, des tirages sur ventes futures ,... équivalents à une année de cargaisons d'avance.

Or, plus de 32 flux financiers dont $25 \%$ sont en nature et $75 \%$ en numéraire, circulent entre les entreprises pétrolières et l'Etat selon un circuit complexe qui pose le problème de la traçabilité des informations liée à l'opacité des transactions (Tableau nº10).

En 2016, le Réconciliateur a effectué des rapprochements sur 1,6 MDS \$, soit plus de 28\% des revenus (hors dons) du Principal et 99,9\% de recettes qu'il a déclaré avoir perçu de 24 entreprises du secteur des hydrocarbures. Les écarts résiduels issus des rapprochements ne sont pas significatifs parce qu'inférieurs au seuil de signification de $5 \%$ de la contribution totale déclarée pour le secteur (Tableau n ${ }^{\circ} 11$ ). Entre 2007 et 2009, les droits du Principal ont représenté $48 \%$ de la production de pétrole, les encaissements correspondants $40 \%$, et les versements nets au Trésor 38\%. L'écart entre les encaissements et versements au Trésor est de $2 \%$. En 2014, ces droits sont de $28 \%$ des revenus totaux inscrits et $99,9 \%$ de ce que le Principal a déclaré avoir perçu. L'écart 0,01est inférieur au seuil de signification de 5\% ;

- la redevance minière proportionnelle pour les hydrocarbures liquides selon le Titre $10 \mathrm{du}$ Code des Hydrocarbures, notamment en ses articles 40 et 53. En 2013, le montant déclarés par les entreprises et celui figurant dans les livres du Trésor fait état d'un manque à gagner de $128 \mathrm{KBbl}$ en 2013 contre moins $5104 \mathrm{KBbl}$ de pétrole. Les sommes encaissées par le Trésor sont inférieures à celles déclarées par les entreprises ;

- le bonus de signature de contrat constitue une prime ou un droit d'entrée, versé à l'Etat en volume d'hydrocarbure pour la conclusion des contrats pétroliers. Dans leur principe, chaque permis fait l'objet d'une comptabilité séparée, sans qu'il y ait de consolidation des pertes et profits entre permis de recherches distincts. L'écart est nul en 2013 et 2014. Cela signifie que les sommes déclarées par les entreprises sont identiques à celles déclarées par les entreprises. Durant la même période, l'écart nul est observé pour le Profil-oil ;

Tableau $n^{\circ} 10$ : Circuit des flux financiers 


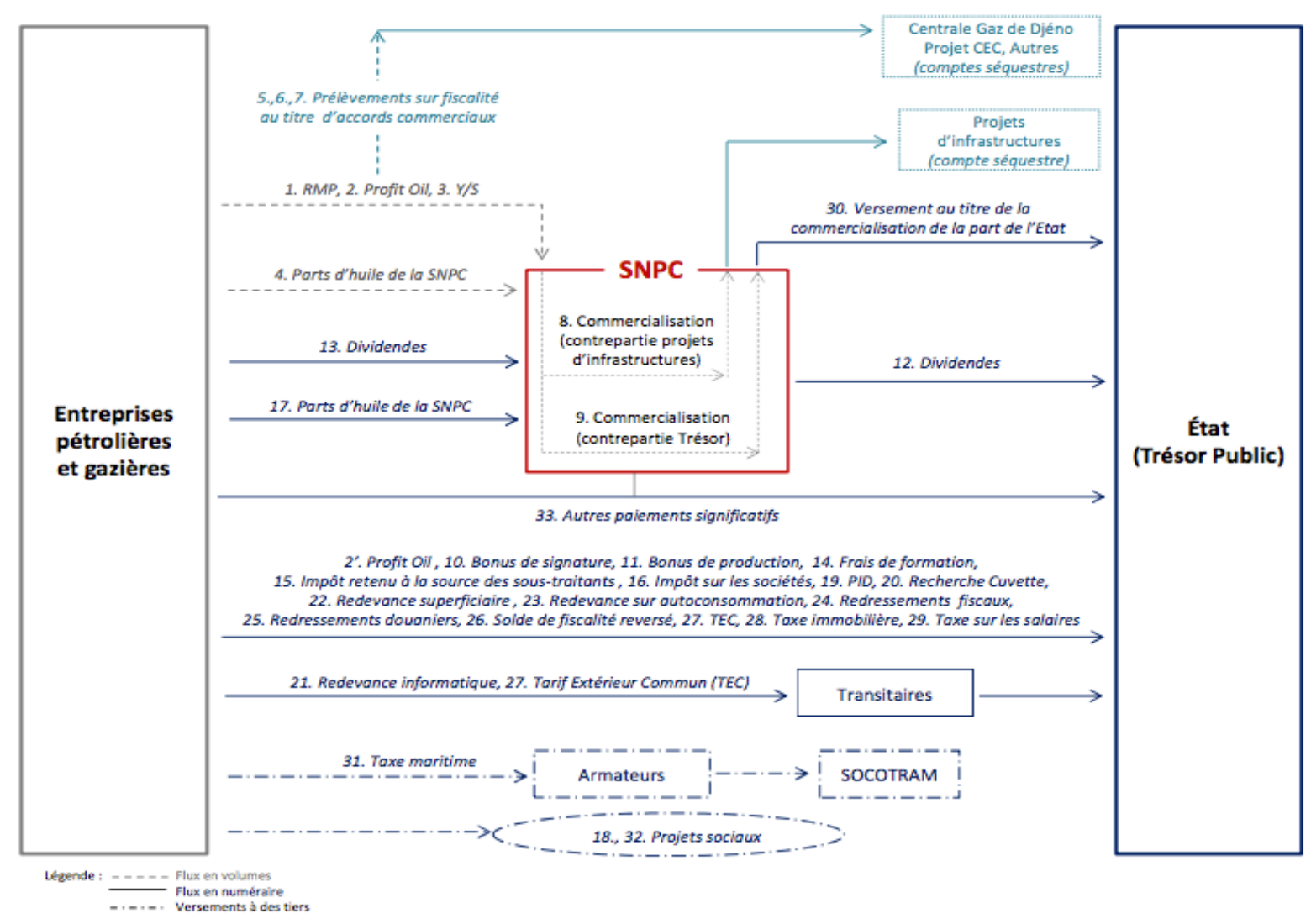

Source : Rapport ITIE 2016

- la provision pour investissements diversifiées (PID) qui a pour objet de permettre d'affecter des fonds au développement de l'économie congolaise. Son montant annuel est de $1 \%$ de la valeur au prix fixé de la production nette de la zone de permis. En 2013 et 2014, les sommes encaissées par le Trésor sont inférieures à celles déclarées par les entreprises.

Les pertes totales liées à l'écart entre les sommes perçues par le Trésor Public et les déclarations des entreprises de la production est passée de 211,83 M \$ en 2004 à 424 M \$ à 2016 (Tableau $\mathrm{n}^{\circ} 12$ ).

III-3- Les causes des écarts : Les écarts constatés ont trois origines :

- la faible application du principe comptable de séparation des exercices (Cut-off): Lors de la clôture annuelle des comptes, des retraitements extracomptables sont effectués afin de rattacher les charges et les produits à l'exercice de référence. Or, le Trésor tient une comptabilité de Caisse ou en partie simple qui enregistre les recettes à la date du jour d'encaissement sans contrepartie. Cette Comptabilisation des recettes réduit la traçabilité des opérations entre les recettes encaissées en $\mathrm{N}$ et celles correspondant à l'exercice $\mathrm{N}-1$. Cela explique les écarts positifs des recettes de 2007 à 2009. La séparation des exercices permettra d'améliorer l'encaissement des recettes selon le principe de la « partie double » qui assure une meilleure traçabilité des opérations. Ce dernier principe signifie que chaque opération à une origine et une destination. Il favorise la transparence des déclarations et des comparaisons des données dans le temps ;

Tableau $n^{\circ} 11$ : Les écarts entre les déclarations des Agents et celles du Principal de 2007 à 2014 


\begin{tabular}{|c|c|c|c|c|c|}
\hline & 2007 & 2008 & 2009 & 2013 & 2014 \\
\hline \multicolumn{6}{|l|}{ Redevance } \\
\hline Etat & 285514234,00 & 341257671,00 & 221413895,00 & 11801000,00 & 12496000,00 \\
\hline Sociétés pétrolières & 185348741,00 & 303648075,00 & 182908203,00 & 11673000,00 & 17600000,00 \\
\hline Ecart en valeur & 100165493,00 & 37609596,00 & 38505692,00 & 128000,00 & $(5104000,00)$ \\
\hline Ecart en $\%$ & $35,08 \%$ & $11,02 \%$ & $17,39 \%$ & $1,08 \%$ & $-40,85 \%$ \\
\hline \multicolumn{6}{|l|}{ PID } \\
\hline Etat & 35754095,00 & 49115991,00 & 35628423,00 & 62412000,00 & 60062000,00 \\
\hline Sociétés pétrolières & 31481903,00 & 42177540,00 & 31442465,00 & 62919000,00 & 60315000,00 \\
\hline Ecart en valeur & 4272192,00 & 6938451,00 & 4185958,00 & $(507000,00)$ & $(253000,00)$ \\
\hline Ecart en $\%$ & $11,95 \%$ & $14,13 \%$ & $11,75 \%$ & $-0,81 \%$ & $-0,42 \%$ \\
\hline \multicolumn{6}{|l|}{ Bonus de signature } \\
\hline Etat & 13491208,00 & - & 3081026,00 & 61088000,00 & 37142000,00 \\
\hline Sociétés pétrolières & 3000000,00 & 500000,00 & 4000000,00 & 61088000,00 & 37142000,00 \\
\hline Ecart en valeur & 10491208,00 & $(500000,00)$ & $(918974,00)$ & - & - \\
\hline Ecart en $\%$ & $77,76 \%$ & $-100,00 \%$ & $-29,83 \%$ & $0,00 \%$ & $0,00 \%$ \\
\hline \multicolumn{6}{|l|}{ Profil-oil } \\
\hline Etat & 410449,00 & 3919258,00 & - & 113000000,00 & 33864000,00 \\
\hline Sociétés pétrolières & 2611368,00 & 1763188,00 & 115189,00 & 113000000,00 & 33577000,00 \\
\hline Ecart en valeur & $(2200919,00)$ & 2156070,00 & $(115189,00)$ & - & 287000,00 \\
\hline Ecart en\% & $-536,22 \%$ & $55,01 \%$ & $-100,00 \%$ & $0,00 \%$ & $0,85 \%$ \\
\hline \multicolumn{6}{|l|}{ Total } \\
\hline Etat & 335169986,00 & 394292920,00 & 260123344,00 & 248301000,00 & 143564000,00 \\
\hline Sociétés pétrolières & 222442012,00 & 348088803,00 & 218465857,00 & 248680000,00 & 148634000,00 \\
\hline Ecart en valeur & 112727974,00 & 46204117,00 & 41657487,00 & $(379000,00)$ & $(5070000,00)$ \\
\hline Ecart en $\%$ & $33,63 \%$ & $11,72 \%$ & $16,01 \%$ & $-0,15 \%$ & $-3,53 \%$ \\
\hline
\end{tabular}

Source : d'après Rapport du Réconciliateur Indépendant 2010 et Rapport d'audit de Finergies 2016 
- le risque de change : Une des sources de différences entre les versements en barils des Contractants et les paiements encaissés par le Trésor provient des différentes étapes suivies par les paiements. Entre le paiement en Baril valorisé en dollars à un instant $\mathrm{T}$ au prix fiscal négocié, la conversion en euros au niveau des banques commerciales des Contractants puis de la SNPC et le transfert en Franc CFA sur le compte du Principal à la Banque Centrale, les commissions et les variations des taux de change altèrent sensiblement le niveau des montants encaissés ;

- la faible traçabilité des données : les livres et les écritures comptables du Contracteur se rapportant aux Travaux Pétroliers sont soumis à l'inspection périodique du Principal. Ils sont tenus en langue française et libellés en dollars et permettent de déterminer la quote-part des Coûts Pétroliers et de la production revenant à chaque partie. Après avoir prévenu le Contracteur par écrit, le Principal exerce ce droit de vérification, pour un exercice donné, soit en faisant appel à son personnel, soit en faisant appel à un cabinet d'audit indépendant internationalement reconnu, désigné par lui et agréé par le Contracteur.

Pour cela, le Principal dispose d'un délai de 15 mois à compter de la date de dépôt des comptes définitifs par le Contracteur pour effectuer en une seule fois, ces examens et vérifications. Les frais afférents à cette vérification sont pris en charge par le Contracteur, dans la limite d'un montant moyen $50.000 \$$ par an, évalué sur une période de deux ans, et actualisé chaque année. Cette dépense fait partie des Coûts Pétroliers.

Tableau ${ }^{\circ} 12$ : Les écarts entre les déclarations des recettes des Agents et celles du Principal de 2004 à 2016 (en millions \$)

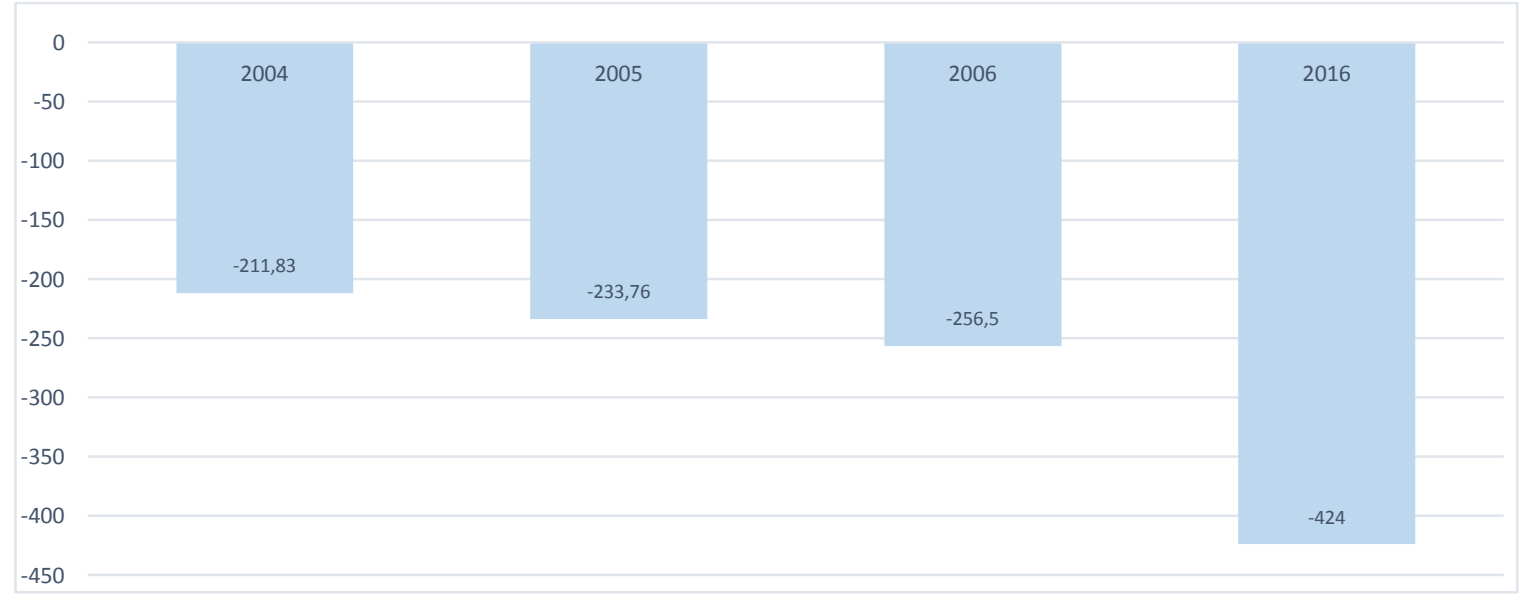

Source : d'après Rapport du Réconciliateur Indépendant 2010 et Rapport d'audit de Finergies 2016

Lamotte et Porcher (2011) qui ont analysés 15 contrats d'exploitation de gisements pétroliers du Congo de 1970 à 2000 ont noté que : "le Congo n'est pas maître des prix de ses ressources. Il ne maîtrise ni le prix mondial - le volume de la production congolaise ne lui permet pas-, ni le prix du pétrole congolais, car les compagnies pétrolières pratiquent le secret sur les prix auxquels elles vendent à leurs sociétés mères ou à des sociétés sœurs. Les prix de vente du pétrole congolais sont donc des prix de transfert, des prix comptables. La commercialisation réalisée par la société nationale SNPC ne permet pas non plus à l'Etat congolais d'accéder à une information précise sur le prix de vente ». Les différents rapports d'audit des cabinets et celui du Réconciliateur Indépendant n'émettent pas de réserves sur la qualité des informations et sur la sincérité des écritures; alors qu'ils constatent une asymétrie informationnelle jugée sans conséquence au seuil inférieur à 5\% des recettes encaissables par le Principal. 
Or, en février 2016, la Direction Financière d'ENI Congo a adressé au Ministère des Hydrocarbures, l'état mensuel de la part des hydrocarbures du Congo sur lequel, les données concernant le Profit-oil et l'Excess-oil mensuels sont fournies globalement (cf annexe $n^{\circ} 1$ ). La contribution de cette entreprise est négative de 35.883,554 Barils, alors qu'elle est positive pour les autres Contracteurs. Le prix unitaire par catégorie de pétrole, les quantités produites, les taux du Cost-oil et du partage de la production des différents gains, n'apparaissent pas sur ce document.

L'absence de ces informations dans la remontée des documents comptables et financiers de l'Agent vers le Principal, conduit ce dernier à faire appel aux auditeurs externes pour établir la sincérité des écritures et des comptes. Cette procédure prévue dans la norme 4.1.C. de l'ETIE évite sans doute des poursuites judiciaires prévues par les articles 64 et suivants du code des hydrocarbures qui punissent d'une amende de 6.000.000 à 200.000.000 FCFA et un emprisonnement de 2 mois à 5 ans ou de l'une de ces deux peines seulement, tout Contracteur auteur de fausse déclaration et de falsification des données relatives à la production et aux impôts et taxes directes et indirectes. Les écarts non expliqués qui constituent en principe des détournements de la production, passent en pertes et profits parce qu'inférieurs au seuil de signification.

\section{Conclusion}

L'objet de cet article était de montrer l'intérêt de la théorie de l'agence dans l'analyse des contrats asymétriques dans lesquels, les comportements opportunistes des agents, limitent la performance du système de gestion et du contrôle budgétaire des régimes fiscaux pétroliers. Le cas étudié montre que la faible traçabilité de l'information et l'absence d'un modèle pertinent d'évaluation de la rente pétrolière entre les parties, rendent difficile, la fixation des prix de transferts et l'optimisation du partage de la rente dans le jeu de coopération.

La constitution des consortiums expose le Principal au risque de sélection adverse, dans la mesure où les entreprises les moins offrantes qu'il sélectionne s'associent aux entreprises les plus offrantes et lui font perdre, la rente informationnelle qu'il aurait gagnée, en sélectionnant l'entreprise la moins efficace. Les entreprises opératrices étant majoritairement les plus réputées du marché, conduisent le Principal à sélectionner les contrats les moins rentables l'exposent au risque d'aléas moral. Au risque systémique lié à la variation des cours de pétrole sur le marché mondial, s'ajoute le risque opérationnel lié à la négociation du prix de transfert entre le Principal et les Agents.

Dans ces conditions, l'application de la norme 4.1.C. de l'ITIE a réduit l'asymétrie de l'information, alors que la faible formalisation du reporting comptable et financier, de la traçabilité des données et l'absence d'un modèle pertinent d'optimisation des gains, accroissent l'incertitude et limitent la rationalité des parties, en termes de moyens et d'action. Le prix fiscal négocié du Baril de pétrole ne permet aux parties d'atteindre des gains optimaux et elles doivent se contenter des gains satisfaisants.

D'où, en situation d'asymétrie informationnelle, la maîtrise des risques des régimes fiscaux pétroliers dépend du degré de traçabilité de l'information, de la pertinence du modèle d'évaluation des gains que disposent les parties et de leur pouvoir de contrôle des termes de la négociation des contrats. 


\section{Bibliographie}

Boistel P. (2008): «La réputation d'entreprise: un impact majeur sur les ressources de l'entreprise, Management et Avenir n ${ }^{\circ} 17$, p.p.9-25

Fama WW., Jensen M.C. (1983): «Separation of Ownership and Control», Journal of Law and Economics 26, June 1983, p $301-326$.

Gruchy A.G. (1972): Contemporary Economic Thought: The Contribution of NeoInstitutional Economics, Clifton, NJ, Augustus M. Kelley, 1972

Jamet P. (1908): La garantie d'intérêt et les grandes compagnies françaises de chemins de fer, Thèse, faculté de Droit de l'université de Bordeaux, Cadoret, 339 p.

Lamotte O., Porcher L. (2011): "Stratégie des compagnies pétrolières internationales et partage de la rente : le cas du Congo », Management et Avenir n ${ }^{\circ} 42$, p-p.310-327

Leenhardt B. (2005), "Fiscalité pétrolière au sud du Sahara : la répartition des rentes », Afrique Contemporaine, $\mathrm{n}^{\circ} 216$, p-p. 65-85.

Jensen M., Meckling W. (1976): «Theory of the firm: managerial behavior, agency cost and ownership structure », Journal of Financial Economics, 3,1976, p-p. 305-360

Ikama J.J (2013): Comment partager la rente pétrolière?: Les enseignements d'une expérience d'une expérience africaine. Ed. Technip, Paris, 2013

Numa G (2009): Théorie de l'agence et concessions de chemins de fer français au $19^{\text {ème }}$ siècle, Revue d'Economie Industrielle, $\mathrm{n}^{\circ} 125$, p-p.105-128

Powell W. et DiMaggio P. (1991): The New Institutionalism in Organizational Analysis, Chicago : University of Chicago Press

Simon H.A. (1982): «Rational decision making in business organization», SIMON H.A: Models of bounded rationality (2 vol.), Cambrige, Mass, The MIT Press, pp. 474-494 (1978 Nobel lecture).

\section{Rapports d'audit :}

Porcher T. (2003) : Mission d'étude relative à l'analyse des ressources pétrolières et des possibilités de leur utilisation pour le financement de la lutte contre la pauvreté en République du Congo, Rapport dans le cadre du programme d'appui de l'Union Européenne au Ministère du Plan de la République du Congo, Brazzaville.

Gherber \& Giurdon (2010) : Rapport du conciliateur indépendant sur les recettes pétrolières de la République du Congo, en 2004-2005-2006, Cabinet d'Avocats, Paris

KPMG (2013) : Rapport de mission d'informations relatives aux encaissements des revenus pétroliers sur les comptes du Trésor de la République du Congo sur la base des procédures convenues. Période du 1er octobre au 31 décembre 2013

Finergies (2016) : Rapport ITIE-Congo, 2016 
Annexe $n^{\circ}$ 1: Fiche mensuelle de la Part de la Production du Congo en Février 2016

\begin{tabular}{|c|c|c|c|c|c|c|c|}
\hline \multirow[b]{2}{*}{ Eléments } & \multicolumn{7}{|c|}{$\begin{array}{l}\text { Contribution des } \\
\text { Contracteurs en Barils de } \\
\text { Pétrole }\end{array}$} \\
\hline & ENI & Total & SNPC & Kontinent & AOGC & New Age & Total \\
\hline Redevance Loango & 13878,763 & 13878,763 & 3265,591 & 1632,796 & & & 32655,913 \\
\hline Redevance Zatchi & 17085,115 & 9199,678 & 4638,493 & & & & 30923,286 \\
\hline Redevance Ikalou (100) & 25101,508 & & & & & & 25101,508 \\
\hline Redevance Nene & 19707,904 & & 3031,985 & & & 7579,963 & 30319,852 \\
\hline Redevance Litchendjili & 2964,131 & & 456,020 & & & 1140,050 & 4560,201 \\
\hline Redevance Kitina II & 4504,562 & & 3291,795 & & 866,261 & & 8662,618 \\
\hline Redevance Djambala II & 1399,355 & & 1119,484 & & 279,871 & & 2798,710 \\
\hline Redevance Foukanda II & 11020,463 & & 6460,272 & & 1520,064 & & 19000,799 \\
\hline Redevance Mwali II & 11903,135 & & 6977,700 & & 1641,812 & & 20522,647 \\
\hline $\begin{array}{l}\text { Redevance Png(Tchibouela/Tchib- } \\
\text { Est/Yanga.S }\end{array}$ & 25722,119 & & & & & & 25722,119 \\
\hline Redevance Tchendo II & 5102,299 & & & & & & 5102,299 \\
\hline Redevance Likouala & 28180,037 & & & & & & 28180,037 \\
\hline Redevance Litanzi & 1228,604 & & & & & & 1228,604 \\
\hline Redevance Tchibeli & 1651,409 & & & & & & 1651,409 \\
\hline Redevance Kombi-Likalala-Libondo & 24332,284 & & & & & & 24332,284 \\
\hline Redevance Awa Paloukou & 17004,888 & & 1889,432 & & & & 18894,320 \\
\hline Redevance Mboundji(100\%) & 63699,015 & & & & & & 63699,015 \\
\hline Redevance Kouakouala (100\%) & 4287,145 & & & & & & 4287,145 \\
\hline Redevance Zingala (100\%) & 6311,907 & & & & & & 6311,907 \\
\hline Redevance Loufika (100) & 181,229 & & & & & & 181,229 \\
\hline Total Redevance (I) & 285265,872 & 23078,441 & 31130,772 & 1632,796 & 4308,008 & 8720,013 & 354135,902 \\
\hline Profil-oil Loango & 11565,636 & 11565,636 & 2721,326 & 1360,663 & & & 27213,261 \\
\hline Profil-oil Zatchi & 14237,596 & 7666,398 & 3865,411 & & & & 25769,405 \\
\hline Profil-oil Ikalou (100\%) & 10670,890 & & & & & & 10670,890 \\
\hline Profil-oil Nene & 7883,161 & & 1212,794 & & & 3031,985 & 12127,940 \\
\hline Profil-oil Litchendjili & 1185,652 & & 182,408 & & & 456,020 & 1824,080 \\
\hline Profil-oil Kitina II & 3753,802 & & 2743,163 & & 721,885 & & 7218,850 \\
\hline Profil-oil Djambala II & 1166,129 & & 932,903 & & 233,226 & & 2332,258 \\
\hline Profil-oil Foukanda II & 12857,206 & & 7536,983 & & 1773,408 & & 22167,597 \\
\hline Profil-oil Mwali II & 13886,991 & & 8140,650 & & 1915,447 & & 23943,088 \\
\hline $\begin{array}{l}\text { Profil-oil Pngl(Tchibouela/Tchib- } \\
\text { Est/Yanga.S }\end{array}$ & 9185,034 & & & & & & 9185,034 \\
\hline Profil-oil Tchendo II & 7866,045 & & & & & & 7866,045 \\
\hline Profil-oil Likouala & 41912,463 & & & & & & 41912,463 \\
\hline Profil-oil Kombi-Likalala-Libondo & 3905,549 & & & & & & 3905,549 \\
\hline Profil-oil Litanzi & 1361,703 & & & & & & 1361,703 \\
\hline Profil-oil Tchibeli & 1830,312 & & & & & & 1830,312 \\
\hline Profil-oil Mboundji (100\%) & 101539,269 & & & & & & 101539,269 \\
\hline Profil-oil Kouakouala (100\%) & 4858,764 & & & & & & 4858,764 \\
\hline Profil-oil Zingali (100\%) & 5785,914 & & & & & & 5785,914 \\
\hline Profil-oil Loufika (100\%) & 220,708 & & & & & & 220,708 \\
\hline Profil-oil Awa Paloukou & 12696,984 & & 1410,776 & & & & 14107,760 \\
\hline $\begin{array}{l}\text { Total Profit-oil (S.P.O. et Excess oil } \\
\text { inclus) (II) }\end{array}$ & 268369,808 & 19232,034 & 28746,414 & 1360,663 & 4643,966 & 3488,005 & 325840,890 \\
\hline Financement CEC-Février 2016 (USD) & $-418519,238$ & & & & & & $-418519,238$ \\
\hline $\begin{array}{l}\text { Récupération des coûts du projet } \\
\text { intégré (USD) }\end{array}$ & $-171000,000$ & & & & & & $-171000,000$ \\
\hline Accords commerciaux (III) & $-589519,238$ & - & - & - & - & - & $=-589519,238$ \\
\hline $\begin{array}{l}\text { Quantité à la disposition de la } \\
\text { République (I+II+III) }\end{array}$ & $-35883,558$ & 42310,475 & 59877,186 & 2993,459 & 8951,974 & 12208,018 & $=90457,554$ \\
\hline PID Février $2016 *$ PFP (\$) & & & & & & & 495788,190 \\
\hline $\begin{array}{l}\text { PID Gaz de Litchendjili Décembre } 2015 \\
(72,22 \%)(\$)\end{array}$ & & & & & & & $+15185,180$ \\
\hline S/Total & & & & & & & $=510973,370$ \\
\hline Frais de Personnel Février 2016 (\$) & & & & & & & $-18385,120$ \\
\hline Total PID Février 2016 * PFP & & & & & & & $=492588,250$ \\
\hline $\begin{array}{l}\text { Total Redevance sur Consommation } \\
\text { Fév.2016* PFP (\$) }\end{array}$ & & & & & & & 18903,300 \\
\hline $\begin{array}{l}\text { Total Redevance-Gaz- Litchendjili- } \\
\text { Février } 16(\$)\end{array}$ & & & & & & & 130362,930 \\
\hline Total à payer (\$) & & & & & & & 641854,480 \\
\hline
\end{tabular}

Source : D’après Direction Financière ENI-Congo, Février 2016 